\section{§27. An Equilibrium Equation of a Magnetized Rotating Plasma}

Saeki, K. (D ept. of Physics, Shizuoka U niv.), Tsushima, A. (D ept. of Physics, Y okohama National Univ.)

Sanuki, $\mathrm{H}$.

The existence of equilibrium solution is one of the most important keys to construct the nuclear fusion devices. The equilibrium of tokamak plasma is sustained by a toroidal current and described by the Grad- Shafranov equation [1]. On the other hand, Stix proposed the plasma confinement by a magnetoelectric torus where the potoidal plasma rotation reduces the charge accumulation.

To get the equilibrium equation, we here start the MHD equations. Then, we employ the momentum balance equation of steady state plasma, the Ohm's law in case of zero resistivity, Poisson equation and the continuity equations for the magnetic field, the electric current density and the plasma flow. Also we treat axisymmetric plasma by using the cylindrical coordinates $r, \vartheta, z$. After the lengthy calculations, we get the GradShafranov equation including the electric field in case of neglecting the convective derivative term, in the form

$$
\begin{aligned}
& \frac{1}{(2 \pi r)^{2}}\left[\frac{1}{\mu_{0}} L \psi+\mu_{0} I \frac{\partial I}{\partial \psi}\right] \\
& +m C_{s}^{2} \frac{\partial n}{\partial \psi}-\varepsilon_{0} \Delta \phi \frac{\partial \phi}{\partial \psi}=0,
\end{aligned}
$$

where $\psi(r, z)$ is the magnetic flux function $\phi$ is the electric charge density and $n$ is the plasma density. In Eq. (1), we used the following notation

$$
L \psi=r \partial / \partial r(\partial \psi / r \partial r)+\partial^{2} \psi / \partial z^{2} .
$$

If we neglect the plasma potential, E1. (1) reduces to the conventional Grad-Shafranov equation describing the tokamak equilibrium. It should be noted that Eq. (1) describes both the tokamak and the magnetoelectric torus at the same time.

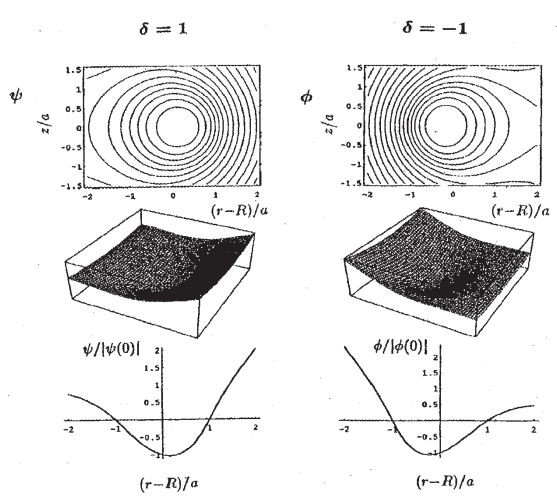

Fig.1 Equilibrium magnetic surface $\psi(r, z)$ of a Tokamak $(\delta=1)$ and equilibrium potential surface $\phi$ of a magnetoelectric torus ( $\delta=-1)$.

We note that $\delta=1$ and $\delta=-1$ means the pure tokamak and the pure magnetoelectric torus, respectively. The change of $\delta$ leads the plasma from the tokamak to the magnetoelectric torus continuously. 